\title{
Association between reduced serum BDNF levels and insomnia with short sleep duration among female hospital nurses
}

\section{AUTHOR(S):}

Furihata, Ryuji; Saitoh, Kaori; Otsuki, Rei; Murata, Sakiko; Suzuki, Masahiro; Jike, Maki; Kaneita, Yoshitaka; Ohida, Takashi; Uchiyama, Makoto

\section{CITATION:}

Furihata, Ryuji ...[et al]. Association between reduced serum BDNF levels and insomnia with short sleep duration among female hospital nurses. Sleep Medicine 2020, 68: 167 172

\section{ISSUE DATE:}

2020-04

URL:

http://hdl.handle.net/2433/245696

\section{RIGHT:}

(c) 2020. This manuscript version is made available under the CC-BY-NC-ND 4.0 license

http://creativecommons.org/licenses/by-nc-nd/4.0/:; The full-text file will be made open to the public on 1 April 2021 in accordance with publisher's 'Terms and Conditions for Self-Archiving'; この論文は出版社版でありません。引用の際に は出版社版をご確認ご利用ください。; This is not the published version. Please cite only the published version. 


\section{Association between reduced serum BDNF levels and insomnia with short sleep duration among female hospital nurses}

Ryuji Furihata ${ }^{\text {ac1 }}$, Kaori Saitoh ${ }^{\mathrm{a}}$, Rei Otsuki ${ }^{\mathrm{a}}$, Sakiko Murata ${ }^{\mathrm{a}}$, Masahiro Suzuki ${ }^{\mathrm{a}}$, Maki Jike ${ }^{\text {bd }}$, Yoshitaka Kaneita $^{\mathrm{b}}$, Takashi Ohida ${ }^{\mathrm{b}}$, Makoto Uchiyama ${ }^{\mathrm{a}}$

a Department of Psychiatry, Nihon University School of Medicine, Tokyo, Japan

b Division of Public Health, Department of Social Medicine, Nihon University School of Medicine, Tokyo, Japan

${ }^{\mathrm{c}}$ Kyoto University Health Services, Kyoto, Japan

${ }^{\mathrm{d}}$ Department of Food Safety and Management, Faculty of Life and Environmental Sciences, Showa Women's University, Tokyo, Japan

${ }^{1}$ These authors contributed equally to this work

The present address of Dr. RF is Kyoto University Health Services, Kyoto, Japan.

The present address of Dr. MJ is Department of Food Safety and Management, Faculty of Life and Environmental Sciences, Showa Women's University, Tokyo, Japan.

Correspondence to: Makoto Uchiyama M.D., Ph.D., Department of Psychiatry, Nihon University School of Medicine, Oyaguchi-kamicho, 30-1 Itabashi-ku, Tokyo 173-8610, Japan.

Tel: +81 3-3972-8111 (ext. 2431)

Fax: +81 3-3972-2920

E-mail: uchiyama.makoto@nihon-u.ac.jp

The total number of figures: 1

The total number of tables: 4

Abstract word count: 242

Word count of manuscript: 2400 


\begin{abstract}
Objective: Previous studies have suggested that brain-derived neurotrophic factor (BDNF) is associated with sleep regulation in humans. However, its relationship with self-reported sleep problems has not been clarified. The aim of the present study was to examine the association between serum BDNF levels and sleep problems among hospital nurses.
\end{abstract}

Methods: Participants were enrolled from among nurses working at a general hospital in Tokyo, Japan. Data from 577 women (age: $35.45 \pm 10.90$ years) were analyzed. This cross-sectional survey was conducted from November to December 2015. Serum BDNF concentrations were evaluated. Participants completed a self-reported questionnaire on sleep including the presence or absence of insomnia symptoms (i.e., difficulty initiating sleep (DIS), difficulty maintaining sleep (DMS), and early morning awakening (EMA)), and sleep duration. Insomnia with short sleep duration (ISS) was defined as: DIS, or DMS, or EMA; and <6 hours sleep duration.

Results: Among 577 participants, 21.3\% reported insomnia, 41.4\% slept less than 6 hours, and finally $12.5 \%$ suffered from ISS. Serum BDNF levels were significantly lower in subjects with ISS than in those without ISS. The serum BDNF levels in insomniacs were significantly lower than in non-insomniacs for short sleep duration $(<6 \mathrm{~h})$, while serum BDNF levels did not differ between insomniacs and non-insomniacs for normal sleep duration ( $\geq 6 \mathrm{~h})$.

Conclusion: This is the first documented study to indicate that ISS is associated with reduced serum BDNF levels. These results may lead to clarification of the underlying pathophysiological relationship between BDNF and poor sleep.

Keywords: serum brain-derived neurotrophic factor; insomnia; sleep duration; women; nurse; Japan 


\section{Highlights}

Evidence suggested that brain-derived neurotrophic factor (BDNF) may participate in the sleep regulation.

We examined serum BDNF levels and sleep problems among female hospital nurses and found that serum BDNF levels were significantly lower in subjects with ISS than in those without. Furthermore, the serum BDNF levels of insomniacs were significantly lower than those in noninsomniacs among subjects with short sleep duration ( $<6 \mathrm{~h})$, whereas serum BDNF levels did not differ between insomniacs and non-insomniacs with normal sleep duration ( $\geq 6 \mathrm{~h}$ ).

Our findings may lead to clarification of the underlying pathophysiological relationship between BDNF and poor sleep. 


\section{Introduction}

Brain-derived neurotrophic factor (BDNF) is a member of the neurotrophin family of growth factors, playing an integral role in neuronal growth and survival, serving as a neurotransmitter modulator, and contributing to neuronal plasticity [1]. BDNF protein and mRNA have been detected in various regions of the brain, and evidence suggests that the level of circulating BDNF (i.e. that in plasma or serum) may reflect the BDNF level in the brain [2]. Most previous studies have suggested that the serum BDNF level is significantly decreased in patients with mental illnesses such as depression [3], bipolar disorder [4], schizophrenia [5], and cognitive decline [6]. Therefore, it has been suggested that serum BDNF is a biomarker of poor mental health.

Several previous studies have suggested that BDNF may participate in the regulation of human sleep. Two experimental studies involving healthy subjects have indicated that the BDNF Val66Met genotype predicted individual variations in polysomnographic features $[7,8]$. More recently, we showed that the serum BDNF level and BDNF Val66Met polymorphism in healthy young adults were associated with the sleep pattern on weekends but not with that on weekdays [9]. A study of patients with different sleep disorders and controls concluded that disturbed sleep due to insomnia, restless legs syndrome or periodic limb movement was associated with decreased serum BDNF levels [10]. However, another study reported that there was no significant difference in BDNF levels among subjects with or without different disorders such as primary insomnia, restless legs syndrome, narcolepsy or idiopathic hypersomnia [11]. Two small-scale polysomnography (PSG) studies conducted in clinical settings have reported that some objective sleep measures were associated with serum BDNF levels, although the results were inconclusive $[12,13]$.

However, the mechanisms underlying the possible relationships between BDNF and sleep in those previous studies were obscure because sleep symptomatology or disorders were not properly controlled for. Furthermore, few studies have examined actual insomnia symptoms, i.e. 
difficulty initiating sleep (DIS), difficulty maintaining sleep (DMS), or early morning awakening (EMA), or sleep duration, which are common components of severe sleep disturbance. Since recent studies have indicated that insomnia with short sleep duration (ISS) is the most biologically severe phenotype of sleep disturbance $[14,15]$, focusing on this phenotype may lead to clarification of the underlying pathophysiological relationship between BDNF and sleep.

Here, we studied the association between the serum BDNF level and sleep problems in a large sample of hospital nurses. The aim of the study was to investigate the association between the serum BDNF level and sleep problems. Our hypothesis was that the serum BDNF level may show differences in association with the various types of sleep problems.

\section{Methods and Materials}

\subsection{Study participants and data collection}

This cross-sectional survey was carried out from November to December 2015 as we have reported previously in detail [16]. Participants were enrolled from among nursing staff working at a general hospital with over 1000 beds in Tokyo, Japan. The target general hospital was affiliated with a medical college. The survey comprised two parts: (1) a self-reported questionnaire on sleep, mental health and lifestyle factors, and (2) examination of blood samples. The director of nursing explained the purposes of the present study and requested the cooperation of the person responsible for each ward of the hospital, who in turn asked for the cooperation of his or her subordinate nursing staff. The distribution and collection of self-reported questionnaires was performed through the director of nursing. Among the participants of the survey (1), those who agreed to participate (2) were subjected to blood testing. Surveys (1) and (2) were conducted within 30 days. All those who had participated in survey (2) provided written informed consent to participate in the study.

A total of 1042 nurses were selected for this complete survey. The number of total participants was 766, and the collection rate was 73.5\%. Among the participants of both surveys 
(1) and (2) (n=618), given their small numbers, men $(n=29)$ were not included. Also, those for whom data on age $(n=1)$ and self-reported sleep ( $n=11)$ were missing were excluded from the present analyses. Finally, we analyzed data from 577 subjects. The study was approved by the ethics committee of Nihon University School of Medicine.

\subsection{Measurement of serum BDNF levels}

Blood samples were taken at AM 7:30-AM 12:00 and centrifuged immediately after blood had been drawn. Serum samples were stored at $-80^{\circ} \mathrm{C}$ until use. BDNF concentration was measured by enzyme-linked immunosorbent assay, using a kit (Quantikine®ELISA, R \& D Systems, Inc.) in accordance with the manufacturer’s instructions [9].

\subsection{Measures of sleep characteristics}

The following self-report questions about sleep characteristics were included in the questionnaire. The questions were created with reference to the questionnaires used in past surveys $[17,18]$.

1. Do you have difficulty falling asleep? (never/seldom/sometimes/often/always): difficulty initiating sleep (DIS)

2. Do you wake up frequently at night? (never/seldom/sometimes/often/always): difficulty maintaining sleep (DMS)

3. Do you wake up too early in the morning? (never/seldom/sometimes/often/always): early morning awakening (EMA)

4. On average, how many hours do you sleep? (Sleep duration): sleep duration

For questions 1-3, participants who answered "often," or "always" were classified as having symptoms. "Insomnia" were defined as the presence of at least one of the sleep-related complaints of DIS, DMS, or EMA based on the definitions used in the previous studies [19, 20].

For question 4, participants who answered "less than 6 hours" were categorized as having "short 
sleep duration”, so as to be consistent with the previous study [21].

ISS was defined as: DIS, or DMS, or EMA; and $<6$ hours sleep duration.

\subsection{Other variables}

The questionnaire included questions designed to obtain sociodemographic information (age, sex), and data on drinking, current smoking, exercise, perceived stress, and shift work. Shift work was divided into two groups: 1/months or less and 2-3/months or more.

\subsection{Statistical analyses}

Categorical variables were expressed as proportions, while continuous variables were expressed as mean and standard deviation (SD). The association between serum BDNF levels and behavioral characteristics or sleep problems were compared using the independent sample t-test, or analysis of variance (ANOVA) for continuous variables (with post hoc tests for significant Fstatistic). A three-way ANOVA was used to examine the main effects of multiple factors and their interactions with serum BDNF levels. All analyses were performed using SPSS 19.0 for Windows. The level of significance was set at $p<0.05$.

\section{Results}

Table 1 shows the association between serum BDNF levels and the demographic and behavioral descriptive characteristics of the study participants. The mean (SD) age was 35.45 (10.90) (range, 21-64) years. The mean (SD) serum BDNF level was 27233 (6663) (range, 816054000) $\mathrm{pg} / \mathrm{mL}$ and showed a normal distribution as demonstrated by the Kolmogorov-Smirnov test $(p=0.068)$. The serum BDNF levels differed significantly among age groups $(p<0.001)$. Post hoc comparisons indicated that serum BDNF levels were significantly higher in subjects aged 40 years or older than in subjects aged 20-29, and 30-39 years. In addition, subjects who were current smokers had significantly higher serum BDNF levels than non-smokers ( $p=0.027)$. 
Among the 577 participants, 21.3\% reported insomnia, 41.4\% slept less than 6 hours, and $12.5 \%$ suffered from ISS. Serum BDNF levels were significantly lower in subjects with ISS than in those without ISS among the total sample $(p=0.028)$ and aged 40 years or older $(p=0.033)$, while they did not differ with respect to other sleep problems (Table 2).

A three-way ANOVA for serum BDNF levels revealed a significant main effect of ISS ( $p=0.048$ ), but no other significant main effects or interactions were found (Table 3).

Relationships between serum BDNF levels and insomnia symptoms were examined separately in subgroups of subjects with short sleep duration $(<6 \mathrm{~h})$ and those with normal ( $\geq 6 \mathrm{~h}$ ) sleep duration. The serum BDNF levels were significantly lower in insomniacs than in noninsomniacs among subjects with short sleep duration $(<6 \mathrm{~h})(p=0.016)$, while they did not differ between insomniacs and non-insomniacs among those with normal sleep duration ( $\geq 6 \mathrm{~h}$ ) (see Fig. 1).

\section{Discussion}

We studied serum BDNF levels and self-reported sleep problems among female hospital nurses and found that serum BDNF levels were significantly lower in subjects with ISS than in those without. Furthermore, the serum BDNF levels of insomniacs were significantly lower than those in non-insomniacs among subjects with short sleep duration $(<6 \mathrm{~h})$, whereas serum BDNF levels did not differ between insomniacs and non-insomniacs with normal sleep duration ( $\geq 6 \mathrm{~h}$ ). These results may lead to clarification of the underlying pathophysiological relationship between BDNF and poor sleep.

We found that serum BDNF levels differed significantly among age groups, and that they were significantly higher in subjects aged 40 years or older than in those aged less than 40 years. Previous studies have revealed that age affects the serum level of BDNF, although mixed results have been reported. One study of women aged between 18 and 65 years [22] and a study of middle-aged women [23] found an age-related increase in serum BDNF levels, while a study 
of older adults found an age-related decrease in serum BDNF levels [24]. These differences may be explained by age differences in study participants and their sex hormone levels [23].

In the present study, subjects who were current smokers had significantly higher serum BDNF levels than non-smokers. Several previous studies have reported that current smokers have higher serum BDNF levels [25, 26]. The results of the present study are in agreement with these previous findings.

The results of previous studies on the association between serum BDNF levels and sleep problems have varied. A cross-sectional study found that participants with self-reported insomnia symptoms, including those with restless legs syndrome or periodic limb movement, showed significantly decreased serum BDNF levels compared with sleep-healthy controls, and serum BDNF levels were significantly correlated with the severity of insomnia in all participants [10]. On the other hand, one study reported that there was no difference in BDNF levels between clinical diagnostic groups including primary insomnia, restless legs syndrome, idiopathic hypersomnia, and narcolepsy [11]. Two small-scale PSG studies have reported that some objective sleep measures were associated with the serum BDNF level. One reported that short sleep duration was associated with decreased serum BDNF levels among patients with insomnia [12], while the other did not find such association, although deep NREM sleep was negatively correlated with the serum BDNF level [13]. In addition, one study of Japanese workers suggested that gender differences may exist: the serum BDNF levels were significantly associated with dyssomnia assessed by the PSQI (Pittsburgh Sleep Quality Index) in females, but not in males [27]. These difference among the above studies may be explained by difference in gender and sleep measures. To clarify sleep symptomatology, the effects of short sleep duration on insomnia symptoms should be considered. In the present study, we used self-reported sleep measures and demonstrated for the first time that serum BDNF levels were significantly lower in subjects with ISS than in those without ISS, and that the serum BDNF levels in subjects with insomnia were significantly lower than those in non-insomniacs in the short sleep duration group ( $<6 \mathrm{~h}$ ), whereas 
serum BDNF levels did not differ between insomniacs and non-insomniacs in the normal sleep duration group ( $\geq 6 \mathrm{~h})$.

In the present study, age group and current smoking were also significantly associated with serum BDNF levels. However, the results of three-way ANOVA showed that no significant interaction existed among these three variables, suggesting that ISS was independently associated with the serum BDNF level in the present sample.

There are several possible interpretations for the association between the serum BDNF level and ISS. First, ISS may decrease the serum level of BDNF. A previous clinical study reported that sleep deprivation therapy changed the serum BDNF levels in depressed patients [28]. This suggested that the serum BDNF level may be affected by curtailment of sleep duration or by recovery from sleep loss. Second, a low BDNF level may cause ISS. An animal study has demonstrated that BDNF in the brain contributes to the regulation of sleep behavior and promotes NREM sleep [29]. Two experimental studies of humans showed that the BDNF Val66Met genotype is related to polysomnographic features of human sleep [7, 8]. These findings suggest that neurotrophic factors may play a vital role in the regulation of sleep. Third, serum BDNF levels and ISS may reflect a common "third variable" such as mental stress, or unmeasured medical disease.

The strengths of this study include a large sample size. Additionally, this is the first study exploring the association between serum BDNF level and sleep problems in Japanese female hospital nurses. However, there were several limitations to this study. First, the results obtained from a cross-sectional study would not be able to determine a causal relationship, i.e. whether sleep problems precede or result from serum BDMF levels. Second, we used a self-reported questionnaire to assess insomnia symptoms and sleep duration. Although some studies of ISS have used self-reported measures to assess sleep duration [30, 31], a previous study pointed out that polysomnography (PSG) and retrospective questionnaire assessments yield different estimates of sleep duration [32]. To evaluate the ISS more precisely, a future study using PSG 
assessment will be required. Third, the validity and reliability of the self-reported questionnaire used to measure sleep problems have not been evaluated. Also, the definitions of insomnia did not conform to the present clinical criteria (DSM-5 and ICSD-3). Fourth, the present study cohort comprised Japanese women of one hospital, and therefore there may be sampling bias, and the results would not address associations between serum BDNF levels and sleep problems in men or other racial groups. Sixth, there may be confounding factors other than those that were considered in the present study. In the present study, we didn’t assess the clinical diagnosis of depression, bipolar disorder, schizophrenia, and cognitive decline. These could have substantially affected both serum BDNF levels and sleep problems.

\section{Conclusions}

This is the first documented study to indicate that serum BDNF levels are significantly lower in subjects with ISS than in those without, and that serum BDNF levels in insomniacs are significantly lower than in non-insomniacs with short sleep duration $(<6 \mathrm{~h})$, whereas serum BDNF levels were shown not to differ between insomniacs and non-insomniacs with normal sleep duration ( $\geq 6 \mathrm{~h}$ ) among Japanese female hospital nurses. These results may lead to clarification of the underlying pathophysiological relationship between BDNF and poor sleep. To increase the generalizability of these findings, future research in men or other racial groups will be required. 


\section{Acknowledgement}

Masahiro Suzuki is supported by a Research Grant from the Japan Society for Promoting Science and Technology Agency (25861032, 2013-2015). Takashi Ohida is supported by a Research Grant from the Japan Society for Promoting Science and Technology Agency (26460849, 2014-2016). Makoto Uchiyama is supported by Health Science Research Grants from the Ministry of Health, Labor and Welfare of the Japanese Government (H20JUNKANKITOU-IPPAN-002 and H25-JUNKANKITOU-IPPAN-007), by a Research Grant from the Japan Society for Promoting Science and Technology Agency (22591301, 2010-2013, 26507012, 2014-2017), by the Research on Development of New Medical Devices from Japan Agency for Medical Research and Development (AMED), by an Intramural Research Grant ( 29-1) for Neurological and Psychiatric Disorders from the National Center of Neurology and Psychiatry, and by a Health Labor Sciences Research Grant 19FA0901. 


\section{Formatting of funding sources}

Dr Uchiyama reports that his department received research support from: Astellas Pharma, Eisai, Meijiseika Pharma, Mochida Pharmaceutical, MSD, Pfizer, Sanofi, Takeda Pharmaceutical, and Yoshitomiyakuhin Corporation. He has provided consulting services to Janssen Pharma, Kao, Shionogi \& Co., Taisho Pharmaceutical and Takeda Pharmaceutical. Dr Kaneita reports that his department received research support from Eisai Co., Ltd. All other authors declare that they have no conflicts of interest. 
Figure 1. Serum BDNF and insomnia by sleep duration category ( $\geq 6 \mathrm{~h}$ or $<6 \mathrm{~h}$ ).

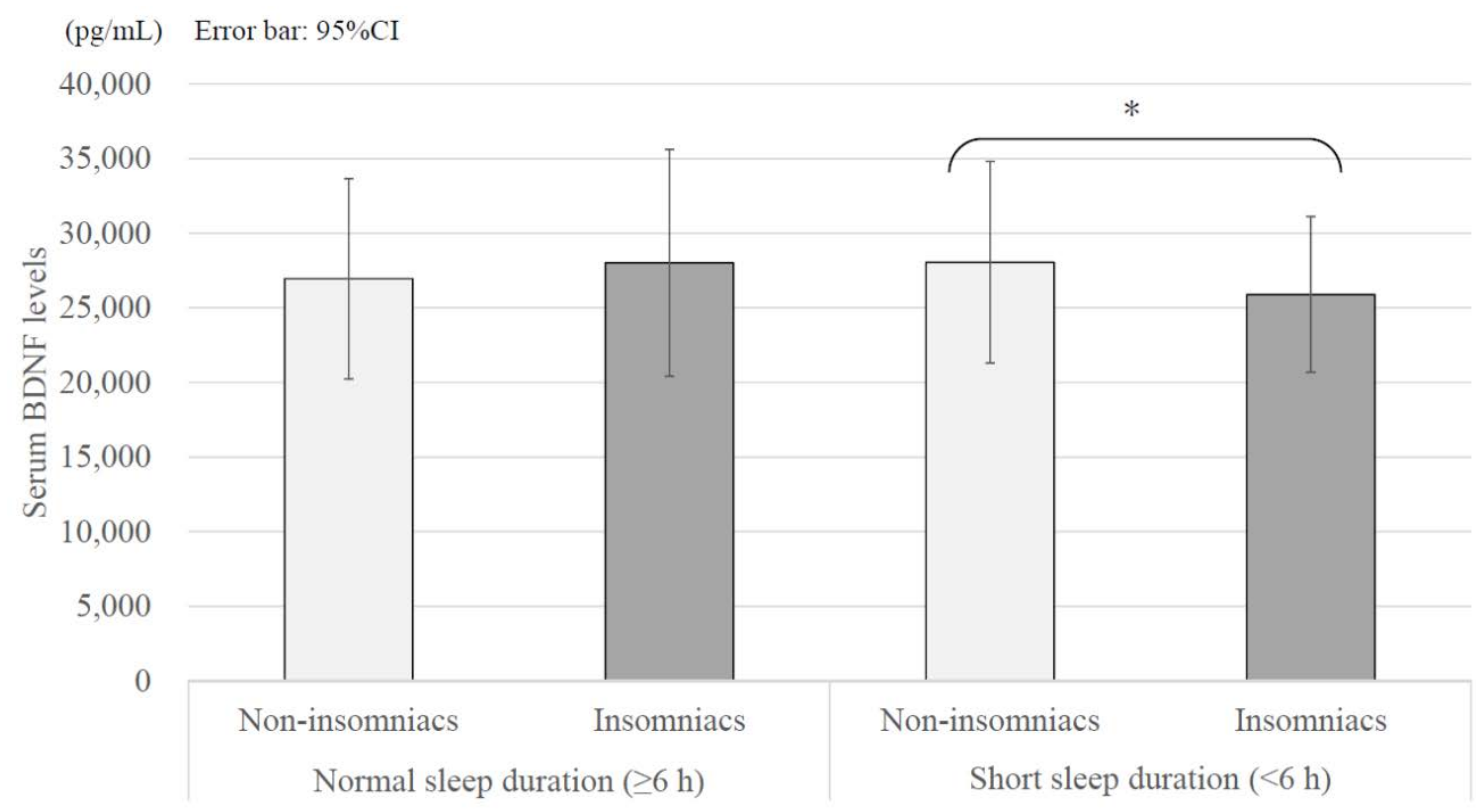

The serum BDNF levels were significantly lower in insomniacs than in non-insomniacs among subjects with short sleep duration $(<6 \mathrm{~h})$, while they did not differ between insomniacs and noninsomniacs among those with normal sleep duration ( $\geq 6 \mathrm{~h}$ ) 
Table 1. The association between serum BDNF levels and demographic and behavioral descriptive characteristics of study participants.

\begin{tabular}{|c|c|c|c|c|c|c|}
\hline & & $\mathrm{N}$ & $\%$ & $\mathrm{~m}$ & SD & $p$-value \\
\hline \multirow[t]{4}{*}{ Age (year) } & & & & & & $<0.001$ \\
\hline & $20-29$ & 217 & 37.6 & 26489 & 6208 & \\
\hline & $30-39$ & 164 & 28.4 & 26086 & 6009 & \\
\hline & $\geq 40$ & 196 & 34.0 & 29015 & 7303 & \\
\hline \multirow[t]{3}{*}{ Drinking } & & & & & & 0.279 \\
\hline & No & 351 & 61.4 & 26965 & 6510 & \\
\hline & Yes & 221 & 38.6 & 27586 & 6905 & \\
\hline \multirow[t]{3}{*}{ Current Smoking } & & & & & & 0.027 \\
\hline & No & 478 & 82.8 & 26954 & 6538 & \\
\hline & Yes & 99 & 17.2 & 28579 & 7117 & \\
\hline \multirow[t]{3}{*}{ Exercise } & & & & & & 0.271 \\
\hline & No & 507 & 88.0 & 27339 & 6579 & \\
\hline & Yes & 69 & 12.0 & 26398 & 7276 & \\
\hline \multirow[t]{3}{*}{ Perceived Stress } & & & & & & 0.749 \\
\hline & No & 430 & 74.5 & 27285 & 6786 & \\
\hline & Yes & 147 & 25.5 & 27080 & 6307 & \\
\hline \multirow[t]{3}{*}{ Shiftwork } & & & & & & 0.453 \\
\hline & $1 /$ Mo or less & 128 & 22.2 & 27691 & 7959 & \\
\hline & 2-3/Mo or more & 448 & 77.8 & 27117 & 6247 & \\
\hline
\end{tabular}

Differences in serum BDNF were examined using the independent sample t-test, or analysis of variance (ANOVA) for continuous variables. 


\section{Table 2. The association between serum BDNF levels and sleep characteristics. (N=577)}

\begin{tabular}{|c|c|c|c|c|c|c|c|c|c|c|c|c|c|c|c|c|c|c|c|c|c|}
\hline & & \multicolumn{5}{|c|}{ Total } & \multicolumn{5}{|c|}{ 20-29y } & \multicolumn{5}{|c|}{ 30-39y } & \multicolumn{5}{|c|}{$>=40 y$} \\
\hline & & $\mathrm{N}$ & $\%$ & $\mathrm{~m}$ & SD & $p$-value & $\mathrm{N}$ & $\%$ & $\mathrm{~m}$ & SD & $p$-value & $\mathrm{N}$ & $\%$ & $\mathrm{~m}$ & SD & $p$-value & $\mathrm{N}$ & $\%$ & $\mathrm{~m}$ & SD & $p$-value \\
\hline \multirow[t]{3}{*}{ Insomnia } & & & & & & 0.391 & & & & & 0.986 & & & & & 0.124 & & & & & 0.745 \\
\hline & No & 454 & 78.7 & 27357 & 6742 & & 168 & 77.4 & 26493 & 6367 & & 133 & 81.1 & 26435 & 6170 & & 153 & 78.1 & 29106 & 7295 & \\
\hline & Yes & 123 & 21.3 & 26775 & 6368 & & 49 & 22.6 & 26476 & 5692 & & 31 & 18.9 & 24587 & 5086 & & 43 & 21.9 & 28693 & 7408 & \\
\hline \multirow[t]{3}{*}{ DIS } & & & & & & 0.434 & & & & & 0.835 & & & & & 0.088 & & & & & 0.904 \\
\hline & No & 483 & 83.7 & 27328 & 6834 & & 177 & 81.6 & 26447 & 6359 & & 140 & 85.4 & 26417 & 6072 & & 166 & 84.7 & 29037 & 7600 & \\
\hline & Yes & 94 & 16.3 & 26740 & 5711 & & 40 & 18.4 & 26675 & 5562 & & 24 & 14.6 & 24154 & 5346 & & 30 & 15.3 & 28897 & 5474 & \\
\hline \multirow[t]{3}{*}{ DMS } & & & & & & 0.143 & & & & & 0.439 & & & & & 0.300 & & & & & 0.466 \\
\hline & No & 527 & 91.3 & 27358 & 6721 & & 203 & 93.5 & 26575 & 6228 & & 145 & 88.4 & 26262 & 6065 & & 179 & 91.3 & 29133 & 7408 & \\
\hline & Yes & 50 & 8.7 & 25912 & 5909 & & 14 & 6.5 & 25243 & 5986 & & 19 & 11.6 & 24737 & 5530 & & 17 & 8.7 & 27778 & 6138 & \\
\hline \multirow[t]{3}{*}{ EMA } & & & & & & 0.347 & & & & & 0.782 & & & & & 0.143 & & & & & 0.630 \\
\hline & No & 526 & 91.2 & 27314 & 6619 & & 200 & 92.2 & 26524 & 6249 & & 151 & 92.1 & 26288 & 6060 & & 175 & 89.3 & 29103 & 7142 & \\
\hline & Yes & 51 & 8.8 & 26395 & 7114 & & 17 & 7.8 & 26088 & 5861 & & 13 & 7.9 & 23738 & 5005 & & 21 & 10.7 & 28287 & 8694 & \\
\hline \multirow[t]{3}{*}{ Sleep duration } & & & & & & 0.600 & & & & & 0.937 & & & & & 0.658 & & & & & 0.728 \\
\hline & $<6 \mathrm{~h}$ & 239 & 41.4 & 27406 & 6394 & & 136 & 62.7 & 26465 & 6863 & & 101 & 61.6 & 26250 & 6063 & & 101 & 51.5 & 28838 & 7321 & \\
\hline & $\geq 6 \mathrm{~h}$ & 338 & 58.6 & 27110 & 6853 & & 81 & 37.3 & 26530 & 4957 & & 63 & 38.4 & 25821 & 5961 & & 95 & 48.5 & 29203 & 7317 & \\
\hline \multirow[t]{3}{*}{ ISS } & & & & & & 0.028 & & & & & 0.687 & & & & & 0.158 & & & & & 0.033 \\
\hline & No & 505 & 87.5 & 27423 & 6827 & & 189 & 87.1 & 26555 & 6336 & & 146 & 89.0 & 26319 & 6051 & & 170 & 86.7 & 29336 & 7573 & \\
\hline & Yes & 72 & 12.5 & 25897 & 5214 & & 28 & 12.9 & 26046 & 5347 & & 18 & 11.0 & 24194 & 5452 & & 26 & 13.3 & 26915 & 4788 & \\
\hline \multirow[t]{3}{*}{ DIS+Short sleep duration ( $<6$ h) } & & & & & & 0.140 & & & & & 0.750 & & & & & 0.160 & & & & & 0.131 \\
\hline & No & 516 & 89.4 & 27373 & 6783 & & 192 & 88.5 & 26538 & 6292 & & 149 & 90.9 & 26295 & 6000 & & 175 & 89.3 & 29208 & 7545 & \\
\hline & Yes & 61 & 10.6 & 26043 & 5445 & & 25 & 11.5 & 26116 & 5622 & & 15 & 9.1 & 24007 & 5898 & & 21 & 10.7 & 27410 & 4647 & \\
\hline & Yes & 29 & 5.0 & 26097 & 5508 & & 9 & 4.1 & 25144 & 6122 & & 10 & 6.1 & 25160 & 6223 & & 10 & 5.1 & 27890 & 4108 & \\
\hline \multirow[t]{3}{*}{ EMA+Short sleep duration $(<6 \mathrm{~h}$ ) } & & & & & & 0.136 & & & & & 0.844 & & & & & 0.164 & & & & & 0.227 \\
\hline & No & 551 & 95.5 & 27322 & 6728 & & 208 & 95.9 & 26507 & 6280 & & 157 & 95.7 & 26224 & 6025 & & 186 & 94.9 & 29162 & 7395 & \\
\hline & Yes & 26 & 4.5 & 25331 & 4776 & & 9 & 4.1 & 26089 & 4457 & & 7 & 4.3 & 22986 & 5061 & & 10 & 5.1 & 26290 & 4770 & \\
\hline
\end{tabular}

Differences in serum BDNF were examined using the independent sample t-test for continuous variables.

DIS, difficulty initiating sleep; DMS, difficulty maintaining sleep; EMA, early morning awakening; ISS, insomnia with short sleep duration (<6h) 
Table 3. The results of three-way ANOVA between serum BDNF levels with ISS, age group, and current smoking (N=577).

\begin{tabular}{|l|c|c|c|}
\hline Factor & DF & \multicolumn{2}{|c|}{ Serum BDNF levels } \\
\hline & & $F$ & $p$ \\
\hline ISS & 1 & 3.935 & 0.048 \\
\hline Age group & 2 & 2.208 & 0.111 \\
\hline Current smoking & 1 & 0.248 & 0.619 \\
\hline ISS $\times$ Age group & 2 & 0.583 & 0.558 \\
\hline ISS $\times$ Current smoking & 1 & 1.016 & 0.314 \\
\hline Age group $\times$ Current smoking & 2 & 0.682 & 0.506 \\
\hline ISS $\times$ Age group $\times$ Current smoking & 2 & 0.270 & 0.763 \\
\hline
\end{tabular}

ANOVA, analysis of variance; DF, degree of freedom; ISS, insomnia with short sleep duration $(<6 \mathrm{~h})$. 
Table 4. The association between serum BDNF levels with insomnia by sleep duration category. (N=577)

\begin{tabular}{|c|c|c|c|c|c|c|c|}
\hline Sleep duration & & & $\mathrm{N}$ & $\%$ & $\mathrm{~m}$ & SD & $p$-value \\
\hline \multirow[t]{12}{*}{ Normal sleep duration ( $\geq 6$ h) } & Insomnia & & & & & & 0.307 \\
\hline & & No & 287 & 84.9 & 26950 & 6715 & \\
\hline & & Yes & 51 & 15.1 & 28014 & 7594 & \\
\hline & DIS & & & & & & 0.418 \\
\hline & & No & 305 & 90.2 & 27011 & 6936 & \\
\hline & & Yes & 33 & 9.8 & 28030 & 6045 & \\
\hline & DMS & & & & & & 0.307 \\
\hline & & No & 317 & 93.8 & 27206 & 6871 & \\
\hline & & Yes & 21 & 6.2 & 25658 & 6554 & \\
\hline & EMA & & & & & & 0.768 \\
\hline & & No & 313 & 92.6 & 27079 & 6680 & \\
\hline & & Yes & 25 & 7.4 & 27501 & 8898 & \\
\hline \multirow[t]{12}{*}{ Short sleep duration (<6 h) } & Insomnia & & & & & & 0.016 \\
\hline & & No & 167 & 69.9 & 28056 & 6751 & \\
\hline & & Yes & 72 & 30.1 & 25897 & 5214 & \\
\hline & DIS & & & & & & 0.054 \\
\hline & & No & 178 & 74.5 & 27873 & 6638 & \\
\hline & & Yes & 61 & 25.5 & 26043 & 5445 & \\
\hline & DMS & & & & & & 0.240 \\
\hline & & No & 210 & 87.9 & 27586 & 6498 & \\
\hline & & Yes & 29 & 12.1 & 26097 & 5508 & \\
\hline & EMA & & & & & & 0.080 \\
\hline & & No & 213 & 89.1 & 27659 & 6528 & \\
\hline & & Yes & 26 & 10.9 & 25331 & 4776 & \\
\hline
\end{tabular}

DIS, difficulty initiating sleep; DMS, difficulty maintaining sleep; EMA, early morning awakening. 


\section{References}

[1] Bathina S, Das UN. Brain-derived neurotrophic factor and its clinical implications. Archives of medical science : AMS. 2015;11:1164-78.

[2] Trajkovska V, Marcussen AB, Vinberg M, Hartvig P, Aznar S, Knudsen GM. Measurements of brain-derived neurotrophic factor: Methodological aspects and demographical data. Brain research bulletin. 2007;73:143-9.

[3] Karege F, Perret G, Bondolfi G, Schwald M, Bertschy G, Aubry JM. Decreased serum brainderived neurotrophic factor levels in major depressed patients. Psychiatry research. 2002;109:1438.

[4] Soares AT, Andreazza AC, Rej S, Rajji TK, Gildengers AG, Lafer B, et al. Decreased BrainDerived Neurotrophic Factor in Older Adults with Bipolar Disorder. The American journal of geriatric psychiatry : official journal of the American Association for Geriatric Psychiatry. 2016;24:596-601.

[5] Green MJ, Matheson SL, Shepherd A, Weickert CS, Carr VJ. Brain-derived neurotrophic factor levels in schizophrenia: a systematic review with meta-analysis. Molecular psychiatry. 2011;16:960-72.

[6] Siuda J, Patalong-Ogiewa M, Zmuda W, Targosz-Gajniak M, Niewiadomska E, Matuszek I, et al. Cognitive impairment and BDNF serum levels. Neurologia i neurochirurgia polska. 2017;51:2432.

[7] Bachmann V, Klein C, Bodenmann S, Schafer N, Berger W, Brugger P, et al. The BDNF 
Val66Met polymorphism modulates sleep intensity: EEG frequency- and state-specificity. Sleep. 2012;35:335-44.

[8] Guindalini C, Mazzotti DR, Castro LS, D'Aurea CV, Andersen ML, Poyares D, et al. Brainderived neurotrophic factor gene polymorphism predicts interindividual variation in the sleep electroencephalogram. Journal of neuroscience research. 2014;92:1018-23.

[9] Saitoh K, Furihata R, Kaneko Y, Suzuki M, Takahashi S, Uchiyama M. Association of serum BDNF levels and the BDNF Val66Met polymorphism with the sleep pattern in healthy young adults. PloS one. 2018;13:e0199765.

[10] Giese M, Unternahrer E, Huttig H, Beck J, Brand S, Calabrese P, et al. BDNF: an indicator of insomnia? Molecular psychiatry. 2014;19:151-2.

[11] Deuschle M, Schredl M, Wisch C, Schilling C, Gilles M, Geisel O, et al. Serum brain-derived neurotrophic factor (BDNF) in sleep-disordered patients: relation to sleep stage N3 and rapid eye movement (REM) sleep across diagnostic entities. Journal of sleep research. 2017.

[12] Fan TT, Chen WH, Shi L, Lin X, Tabarak S, Chen SJ, et al. Objective sleep duration is associated with cognitive deficits in primary insomnia: BDNF may play a role. Sleep. 2019;42.

[13] Mikoteit T, Brand S, Eckert A, Holsboer-Trachsler E, Beck J. Brain-derived neurotrophic factor is a biomarker for subjective insomnia but not objectively assessable poor sleep continuity. Journal of psychiatric research. 2019;110:103-9.

[14] Fernandez-Mendoza J. The insomnia with short sleep duration phenotype: an update on it's importance for health and prevention. Current opinion in psychiatry. 2017;30:56-63. 
[15] Vgontzas AN, Fernandez-Mendoza J, Liao D, Bixler EO. Insomnia with objective short sleep duration: the most biologically severe phenotype of the disorder. Sleep medicine reviews. $2013 ; 17: 241-54$.

[16] Furihata R, Saitoh K, Suzuki M, Jike M, Kaneita Y, Ohida T, et al. A composite measure of sleep health is associated with symptoms of depression among Japanese female hospital nurses. Compr Psychiatry. 2020;97:152151.

[17] Morioka H, Jike M, Kanda H, Osaki Y, Nakagome S, Otsuka Y, et al. The association between sleep disturbance and second-hand smoke exposure: a large-scale, nationwide, cross-sectional study of adolescents in Japan. Sleep medicine. 2018;50:29-35.

[18] Morioka H, Itani O, Kaneita Y, Ikeda M, Kondo S, Yamamoto R, et al. Associations between sleep disturbance and alcohol drinking: A large-scale epidemiological study of adolescents in Japan. Alcohol (Fayetteville, NY). 2013;47:619-28.

[19] Furihata R, Kaneita Y, Jike M, Ohida T, Uchiyama M. Napping and associated factors: a Japanese nationwide general population survey. Sleep medicine. 2016;20:72-9.

[20] Furihata R, Uchiyama M, Suzuki M, Konno C, Konno M, Takahashi S, et al. Association of short sleep duration and short time in bed with depression: A Japanese general population survey. Sleep and Biological Rhythms. 2015;13:136-45.

[21] Furihata R, Uchiyama M, Takahashi S, Konno C, Suzuki M, Osaki K, et al. Self-help behaviors for sleep and depression: a Japanese nationwide general population survey. Journal of affective disorders. 2011;130:75-82. 
[22] Bus BA, Molendijk ML, Penninx BJ, Buitelaar JK, Kenis G, Prickaerts J, et al. Determinants of serum brain-derived neurotrophic factor. Psychoneuroendocrinology. 2011;36:228-39.

[23] Elfving B, Buttenschon HN, Foldager L, Poulsen PH, Andersen JH, Grynderup MB, et al. Depression, the Val66Met polymorphism, age, and gender influence the serum BDNF level. Journal of psychiatric research. 2012;46:1118-25.

[24] Bus BA, Tendolkar I, Franke B, de Graaf J, den Heijer M, Buitelaar JK, et al. Serum brainderived neurotrophic factor: determinants and relationship with depressive symptoms in a community population of middle-aged and elderly people. The world journal of biological psychiatry : the official journal of the World Federation of Societies of Biological Psychiatry. 2012;13:39-47.

[25] Suriyaprom K, Tungtrongchitr R, Thawnashom K, Pimainog Y. BDNF Val66Met polymorphism and serum concentrations of BDNF with smoking in Thai males. Genetics and molecular research : GMR. 2013;12:4925-33.

[26] Jamal M, Van der Does W, Elzinga BM, Molendijk ML, Penninx BW. Association between smoking, nicotine dependence, and BDNF Val66Met polymorphism with BDNF concentrations in serum. Nicotine \& tobacco research : official journal of the Society for Research on Nicotine and Tobacco. 2015;17:323-9.

[27] Nishichi R, Nufuji Y, Washio M, Kumagai S. Serum brain-derived neurotrophic factor levels are associated with dyssomnia in females, but not males, among Japanese workers. Journal of clinical sleep medicine : JCSM : official publication of the American Academy of Sleep Medicine. 
2013;9:649-54.

[28] Gorgulu Y, Caliyurt O. Rapid antidepressant effects of sleep deprivation therapy correlates with serum BDNF changes in major depression. Brain research bulletin. 2009;80:158-62.

[29] Kushikata T, Fang J, Krueger JM. Brain-derived neurotrophic factor enhances spontaneous sleep in rats and rabbits. The American journal of physiology. 1999;276:R1334-8.

[30] Jackowska M, Poole L. Sleep problems, short sleep and a combination of both increase the risk of depressive symptoms in older people: a 6-year follow-up investigation from the English Longitudinal Study of Ageing. Sleep medicine. 2017;37:60-5.

[31] Lin SC, Sun CA, You SL, Hwang LC, Liang CY, Yang T, et al. The Link of Self-Reported Insomnia Symptoms and Sleep Duration with Metabolic Syndrome: A Chinese Population-Based Study. Sleep. 2016;39:1261-6.

[32] Matthews KA, Patel SR, Pantesco EJ, Buysse DJ, Kamarck TW, Lee L, et al. Similarities and differences in estimates of sleep duration by polysomnography, actigraphy, diary, and self-reported habitual sleep in a community sample. Sleep Health. 2017;4:96-103. 